\title{
Nutrition des bovins tropicaux dans le cadre des élevages extensifs sahéliens : mesures de consommation et appréciation de la digestibilité et de la valeur alimentaire des fourrages
}

\author{
$2^{\text {e }}$ Partie : Note concernant les résultats d'une première série \\ de « digestibilité in vivo » sur mouton
}

par S. Diallo, P. L. PUGliese, H. CALVET (**)

\begin{abstract}
RESUME
Les auteurs rapportent les résultats de 39 expérimentations de « digestibilité in vivo » sur mouton de différents types de rations: des fanes d'arachide et de haricot, de la paille de riz, divers foins et fourrages, ainsi que des rations composées. De ces résultats, il ressort que cette méthode d'appréciation de la digestibilité est satisfaisante et qu'elle est d'autant plus efficace que la ration testée est de composition simple et constante.
\end{abstract}

\section{INTRODUCTION}

Les 39 expérimentations dont les résultats font l'objet de cette note entrent dans le cadre du programme intitulé «Etudes des problèmes alimentaires saisonniers qui se posent au niveau des élevages extensifs sénégalais » dont les objectifs" et la méthodologie générale ont déjà fait l'objet d'une première publication (13).

Ce programme, pour l'essentiel, se propose de mesurer la consommation journalière des

(*) L'étude de la nutrition des bovins tropicaux dans le cadre des élevages extensifs sahéliens fait l'objet d'un thème de recherche inscrit au IVe plan de développement économique et social de la République du Sénégal.

(**) Laboratoire national de l'Elevage et de Recherches vétérinaires B. P. 2057, Dakar-Hann, Sénégal. troupeaux au pâturage et d'estimer en même temps la digestibilité des fourrages ingérés aux différentes périodes du cycle annuel tropical.

L'approximation de la digestibilité des fourrages sur le pâturage nécessite la mise au point de méthodes spéciales. Chaque ration expérimentale est donc analysée simultanément par 5 méthodes de digestibilité dont les résultats seront comparés à ceux fournis par l'expérimentation in vivo sur mouton utilisée comme méthode de référence. Mais en dehors de leur rôle d'élément de comparaison, les digestibilités sur mouton présentent par elles-mêmes un intérêt propre : celui de déterminer ou de préciser la valeur alimentaire d'un certain nombre de rations utilisées ou utilisables au Sénégal. C'est ce dernier aspect qui constitue la matière du présent article. 


\section{MATÉRIEL ET MÉTHODES}

La méthodologie utilisée pour les «digestibilités in vivo sur mouton » s'inspire des travaux effectués dans ce domaine par DEMARQUILLY au C. N. R. Z. de Theix. Le mouton a été choisi pour ces tests, parce qu'il présente par rapport aux bovins deux avantages essentiels: d'une part une plus grande maniabilité et une meilleure tenue dans les cages, d'autre part la possibilité de tester une ration avec des quantités d'aliments 10 fois plus faibles. Ces avantages sont d'autant plus appréciables que, comme le soulignent les différents auteurs, les résultats obtenus sur moutons et rapportés au poids métabolique (*) sont directement transposables aux bovins.

\section{II.1. Matériel}

Les cages à métabolisme sont en bois et ont été construites dans les ateliers du Laboratoire. Elles ont $1 \mathrm{~m}$ de long, $46 \mathrm{~cm}$ de large et sont disposées par batteries de 4 sur un support qui les maintient à $70 \mathrm{~cm}$ du sol.

La face antérieure est formée par deux tubes espacés de $9 \mathrm{~cm}$, dont l'un est amovible, ce qui permet en serrant le cou de l'animal, de limiter ses déplacements et de maintenir sa tête dans la caisse à fourrage fixée en avant des cages, et équipée d'un abreuvoir à niveau constant.

Les fèces sont recueillies dans un grand bac en plastique qui coulisse sous la cage.

Les animaux sont pesés sur un pèse-bétail à demeure dans l'étable d'une capacité de $1000 \mathrm{~kg}$, gradué par $100 \mathrm{~g}$.

La ration est pesée sur une bascule monoplateau à grand cachant graduée par $50 \mathrm{~g}$.

Les pesées précises se font soit à la balance monoplateau Mettler, avec dispositif de tare rapide et d'une portée maximale de $2 \mathrm{~kg}$, soit à la balance de précision Mettler type P311. Les fourrages et les fèces sont séchés dans une étuve à $80^{\circ}$, ventilés puis broyés dans un broyeur à marteaux Gondar.

\section{II.2. Les animaux}

Les moutons sont de race Peulh-peulh sénégalaise. Ce sont des animaux de petite taille

(*) Le poids métabolique (Metabolic body size MBS - des auteurs américains) est égal au poids vif élevé à la puissance 0,75 . pesant de 20 à $30 \mathrm{~kg}$, dépourvus de laine et dont les longs poils plus ou moins ondulés sont noirs tachetés de blanc.

Ils sont déparasités à leur entrée dans l'étable de digestibilité et surveillés périodiquement par des examens coprologiques durant les périodes de repos, entre les expériences.

\section{II.3. La méthode}

Chaque unité expérimentale comprend 4 animaux en cages de digestibilité et 6 animaux maintenus en stalle pour des mesures de consommation.

Les fourrages sont distribués en 2 ou 3 repas. Les quantités sont adaptées de façon à obtenir un refus équivalent au $10^{\mathrm{e}}$ de la ration.

Les refus sont récoltés tous les matins avant la première distribution, en même temps que les fèces des 24 h précédentes.

Ces opérations sont concomitantes à la constitution de 3 types d'échantillon :

Le premier correspond au fourrage distribué. Il est constitué en prélevant sur la masse du fourrage à distribuer préalablement bien mélangé plusieurs poignées de façon à obtenir un échantillon de $700 \mathrm{~g}$ à $1 \mathrm{~kg}$, qui est mis à l'étuve pour dessiccation.

Si l'échantillon de fourrage distribué est global et correspond aux 4 animaux, les échantillons de refus et de matières fécales sont individuels. Ceci permet d'enlever du mélange final les refus et les matières fécales d'un individu qui se serait mal comporté durant la période expérimentale.

Chaque matin, les refus sont pesés et desséchés à l'étuve en totalité ou en partie suivant leur volume. Après établissement du taux de matière sèche, ils sont conservés dans des sacs en plastique étiquetés au jour et au numéro de l'animal et conservés jusqu'à la fin de l'expérience.

Le même procédé est appliqué aux matières fécales. La durée de chaque expérimentation est de 14 jours. Les 7 premiers jours servent à l'adaptation des animaux au régime. Les 7 jours suivants, les animaux séjournent dans les cages à métabolisme.

En fin d'expérience, on dispose donc de 7 échantillons desséchés de fourrage distribué, de 28 échantillons desséchés de refus et de 28 échantillons de fèces. 
Les fourrages distribués sont alors finement broyés, homogénéisés dans un mélangeur et vont constituer l'échantillon moyen final qui est envoyé au Laboratoire pour analyse.

Les 28 sachets de refus et de matières fécales subissent le même sort et aboutissent également à un échantillon moyen de refus et un échantillon moyen de matières fécales destinés à être dosés.

\section{4. Analyses chimiques}

Les 3 types d'échantillon sont soum1s aux mêmes dosages qui permettent d'établir les taux de matière sèche, de cellulose brute (méthode de WEENDE), de matières minérales et de protéines brutes (méthode de KJELDAHL).

Les calculs ultérieurs permettent d'établir les résultats définitifs qui intéressent :

a) Les coefficients de digestibilité de la matière sèche, de la matière organique, des matières protéiques, de la matière grasse, de la cellulose et de l'extractif non azoté en appliquant la formule :

coeff. de digest. $=$

$$
=\frac{\text { matières ingérées }- \text { matières excrétées }}{\text { matières ingérées }} \text {. }
$$

b) La valeur fourragère de l'aliment qui est établie suivant la formule classique :

$$
\begin{aligned}
& \mathrm{UF} / \mathrm{kg} \mathrm{MS}= \\
& =\frac{[\mathrm{MAD}+\mathrm{MCD}+\mathrm{ENAD}+(\mathrm{MGD} \times 2,25)]}{\mathbf{3 , 6 5}-\mathrm{MS}}
\end{aligned}
$$

ou suivant les formules de BREIREM exprimées en UF ou en UA (unité amidon)

$$
\begin{aligned}
\mathrm{UF} / \mathrm{kg} \mathrm{MS} & =\frac{2,36 \mathrm{MOD}-1,20 \mathrm{MOND}}{1650} \\
\mathrm{UA} / \mathrm{kg} \mathrm{MS} & =\frac{2,36 \mathrm{MOD}-1,20 \mathrm{MOND}}{2360}
\end{aligned}
$$

- MOD = matière organique digestible

- MOND = matière organique non digestible

c) L'indice des quantités ingérées

Cet indice a pour base 100 et correspond à la moyenne des quantités ingérées pour l'ensemble des échantillons étudiés à Theix, c'est-à-dire à $70 \mathrm{~g}$ par $\mathrm{kg}$ de poids métabolique $\left(\mathrm{P}^{0,75}\right)$.
L'indice des quantités ingérées d'un fourrage est donc calculé de la façon suivante :

$$
\begin{gathered}
\mathrm{IQ}=\text { quantité ingérée de fourrage } \\
\frac{\left(\mathrm{g} \mathrm{MS} / \mathrm{kg} \mathrm{P}^{0,75}\right) \times 100}{70} .
\end{gathered}
$$

Cet indice permet de classer rapidement les fourrages suivant leur appétence les uns par rapport aux autres.

d) L'indice de valeur alimentaire

L'indice de valeur alimentaire est égal au produit de la quantité ingérée et de la concentration en unités amidon du fourrage étudié.

La moyenne des valeurs trouvées à Theix a été de 0,0352. L'indice de valeur alimentaire d'un fourrage sera donc donné par la formule suivante :

$$
\begin{aligned}
& \text { UA } / \mathrm{kg} \text { MS } \times \text { Quantité ingérée } \\
& \text { en } \mathrm{kg} \mathrm{MS} / \mathrm{kg} \mathrm{P}^{0,75} \\
& \mathrm{IVa}=\frac{\times 100}{0,0352} \text {. }
\end{aligned}
$$

Cet indice intégrant à la fois la consommation et la valeur fourragère constitue l'approximation la meilleure de la valeur d'un aliment.

\section{LES RATIONS TESTÉES}

Le tableau I donne la nature des rations utilisées, le nombre de leur répétition, leur numéro d'ordre chronologique. Nous énonçons, par la suite, quelques caractéristiques des fourrages ou rations utilisés au cours de ces expériences.

\section{1. La fane d'arachide}

La fane d'arachide est un fourrage très appété par toutes les espèces animales. Il est constitué par la partie végétative de l'arachide à la suite du battage et de la récolte des gousses. Les disponibilités au Sénégal sont théoriquement importantes puisque la proportion moyenne de fanes par rapport à la gousse est de 1,5. Les $500000 \mathrm{t}$ produites ces dernières années laisseraient $750000 \mathrm{t}$ de fourrage.

Comme le montreront les prochains résultats, l'hétérogénéité de ce produit est très grande.

\section{2. La paille de riz}

La paille de riz est un fourrage abondant dans les régions rizicoles du Sénégal qui sont 
TABLEAU $N^{D} I$, Rations entrant dans le cadre de cetre première sêrie.

\begin{tabular}{|c|c|c|c|}
\hline & Na $t u r e c e$ l'a $11 \mathrm{ment}$ & $\begin{array}{l}\text { Nombre de } \\
\text { répétitions }\end{array}$ & $\begin{array}{l}N^{0} d^{\prime} \text { orcire } \\
\text { chrorologique }\end{array}$ \\
\hline 1 & $\begin{array}{l}\text { - Eare d'arachide passée au gyrobroyeur } \\
\text { - fane d'arachide pulvérulente (passếe au moulin à martesux) } \\
\text { - fane d'arachide pulvérulente + farine de sorgho } \\
\text { - Eane d'arachide gyrobroyée + graine de coton } \\
\text { - fane de harıcots (bUD) }\end{array}$ & $\begin{array}{l}5 \\
1 \\
1 \\
1 \\
1\end{array}$ & $\begin{array}{l}1-2-14-34-35 \\
3 \\
6 \\
10 \\
12\end{array}$ \\
\hline 2 & $\begin{array}{l}\text { - paille de riz brute } \\
\text { - paille de riz + gralnes de coton } \\
\text { - paille de riz trattée à l'urée }\end{array}$ & $\begin{array}{l}3 \\
6 \\
1\end{array}$ & $\begin{array}{l}15-16-17 \\
9-11-13-14-19-=0 \\
8\end{array}$ \\
\hline 3 & $\begin{array}{l}\text { - foin de praurie de Sangalkan } \\
\text { - foin de pranrie de Sangalkam + tourteau d'arachide } \\
\text { - foin de prairie de Sangalkam traité à l'urée } \\
\text { - foin de prairie de Dara }\end{array}$ & $\begin{array}{l}1 \\
1 \\
1 \\
2\end{array}$ & $\begin{array}{l}4 \\
5 \\
7 \\
32-33\end{array}$ \\
\hline 4 & $\begin{array}{l}\text { - foin de Panisum manzmm } \\
\text { - foin ce Pennzetum (variété rizozí) }\end{array}$ & $\begin{array}{l}4 \\
4\end{array}$ & $\begin{array}{l}2]-22-23 \\
24-25-26-27\end{array}$ \\
\hline 5 & - ration composée utilusée en cmbouche en 1973 & 6 & $28-29-30-31$ \\
\hline 6 & $\begin{array}{l}\text { - son de maïs } \\
\text { - son de maîs + tourteau de "bëref" } \\
\text { - foin de Panicum mawimum + tourteau de "bĕref" }\end{array}$ & $\begin{array}{l}1 \\
1 \\
]\end{array}$ & $\begin{array}{l}40 \\
37 \\
42\end{array}$ \\
\hline
\end{tabular}

Le "béref" est le nom local de certanes varcêtés de pastiques (farılle des cucurhitacées) lont les graines après extraction de 1 'huile donnent le tourteau.

Rectificatif : « 2 »dernière colonne, $2^{\mathrm{e}}$ Jigne, lire 18 au lieu de 14.

« 4 » Foin de Panicum, lire 3 au lieu de 4.

essentiellement la région du fleuve, la Casamance, le Sine Saloum et accessoirement le Sénégal oriental.

Sur le fleuve, les statistiques pour l'année 1971 font apparaître une production de $26000 \mathrm{t}$ de Paddy.

En Casamance, la production s'élevait à $60000 \mathrm{t}$ durant la même année tandis qu'au Sine Saloum, elle était de $5000 \mathrm{t}$.

$\mathrm{Au}$ total, à cette date, on récoltait donc au Sénégal plus de $90000 \mathrm{t}$ de paddy, ce qui correspond à environ $150000 \mathrm{t}$ de paille.

Un très faible pourcentage de la récolte est utilisé comme aliment du bétail. Par manque de moyens pour l'enlever, la paille se trouve encore dans les casiers au moment de la remise en culture, et doit être brûlée dans la plupart des cas.

\section{III.3. Plantes fourragères}

Depuis plusieurs années, des cultures fourragères sont pratiquées à la ferme de Sangalkam. A l'heure actuelle, des installations d'arrosage par aspersion à partir de plusieurs forages permettent d'entrer dans une phase de production intensive. Un programme visant à déterminer la productivité, la valeur alimentaire de ces plantes en fonction des espèces, du cycle végétatif et des différentes saisons est en cours de réalisation.
Les études de digestibilité que nous rapportons ici concernent une recoupe de Panicum maximum et de Pennisetum purpureum variété Kizozi exploitées en fin de cycle végétatif alors que pour ces deux espèces, le stade optimal pour la coupe se situe environ à 30 jours.

\section{III.4. Foins de prairies naturelles}

Les deux types de fourrages analysés proviennent de régions très différentes.

Le premier a été fauché et fané sur des parcelles du centre de recherches zootechniques de Dara situé en région sahélienne. Il est à base de graminées fines où domine Zornia glochidiata.

Le second a été récolté dès la fin de la saison des pluies sur des prairies dunaires de la ferme de Sangalkam située à $35 \mathrm{~km}$ de Dakar dans la région des Niayes.

II est d'un aspect beaucoup plus grossier.

\section{III.5. Les rations d'embouche utilisées en 1973}

Les expérimentations d'embouche réalisées en 1973 avaient pour thème général, l'utilisation des sous-produits de l'industrie cotonnière : la graine de coton, la coque vide de graine, le tourteau de coton. 
La ration utilisée dans les expérimentations de digestibilité avait la composition centésimale suivante :

coque d'arachide $\ldots \ldots \ldots \ldots \ldots \ldots 20$

graine de coton $\ldots \ldots \ldots \ldots \ldots \ldots \ldots . \ldots \ldots$

son de mails ................. 25

farine de maïs ............... 25

tourteau d'arachide............. 2

carbonate de chaux............. 1

sel $\ldots \ldots \ldots \ldots \ldots \ldots \ldots \ldots \ldots$ l

Cette ration servie à des taurillons zébu de race Gobra âgés de 3 ans et pesant en moyenne en début d'essai $72 \mathrm{~kg}$, a permis d'obtenir durant 85 jours un gain quotidien moyen de $938 \mathrm{~g}$.

\section{LES RÉSULTATS}

\section{IV .1. Digestibilités directes}

Pour chaque catégorie d'aliment, les résultats comporteront l'analyse bromatologique, les coefficients de digestibilité obtenus, les valeurs fourragères, l'indice de consommation (IQ) et l'indice de valeur alimentaire (IVa),

Les tableaux $n^{\circ}$ II et $n^{\circ}$ JII présentent les résultats moyens obtenus au cours des analyses bromatologiques et des calculs conduisant aux coefficients de digestibilité. Le tableau $n^{\circ}$ IV regroupe les valeurs fourragères des aliments utilisés, les indices de consommation (IQ) et les indices de valeur alimentaire (IVa).

Concernant ces données, il paraît nécessaire de fournir les précisions suivantes :

- le dosage de la lignine est effectué par la méthode de Van Soest qui fait intervenir dans un premier temps un détergent (Cétyl-Triméthyl-ammonium bromide) suiv1 d'une attaque à l'acide sulfurique à $72 \mathrm{p}, 100$ (lignine $\mathrm{l}$ ) ou au permanganate de potassium (lignine 2) ;

- pour la ration $n^{\circ} 6$, la proportion entre la fane pulvérulente et la farine de sorgho est de $3 / 4$ de fane pour $1 / 4$ de sorgho;

- dans la ration 10 , la proportion entre la fane et la graine de coton est de $4 / 5$ de fane pour $1 / 5$ de graines de coton;

- Ia BUD est une entreprise industrielle de maraîchage installée au Sénégal qui produit des légumes à contre-saison pour l'exportation vers l'Europe.
Cette exploitation laisse des quantités importantes de sous-produits, au nombre desquels se trouvent les fanes de haricot.

\section{IV.2. Digestibilités différentielles}

Ce sont des méthodes particulières qui permettent d'obtenir une approximation de la digestibilité de produits alimentaires qui ne peuvent constituer à eux seuls une ration soit en raison de leur composition, soit à cause de leur faible appétence.

Le schéma type de ces digestibilités est le suivant - - soient $\mathrm{A}$ et $\mathrm{B}$ deux composants de ration (B ne pouvant être administré seul à l'animal).

Dans un premier temps, on effectue une expérience de digestibilité avec A qui permet de connaître les coefficients de digestibilité de A.

Une deuxième expérience utilise la ration $\mathrm{A}+\mathrm{B}$ dans des proportions connues. A partir de ces derniers résultats, par différence, et en utilisant les données de la première expérience on obtient les coefficients de B.

Les calculs de ce type ont été utilisés pour apprécier les valeurs de la graine de coton et celles du tourteau de Béref.

\section{IV.2.1. Digestibilité des graines de coton}

Les résultats concernant la graine de coton ont été traités par méthode différenticlle, en partant des digestibilités paille de riz + graıne de coton $\mathrm{n}^{07}$ 18-19-20 et des coefficients moyens obtenus sur la paille de riz: digestibilités $\mathrm{n}^{\mathrm{os}}$ 15-16-17.

La méthode de calcul utilisant les données obtenues dans les cages à métabolısme est présentée dans le tableau $\mathrm{V}$.

La valeur bromatologique de la graine de coton en fonction de ces calculs est la suivante :

$$
\begin{aligned}
& \mathrm{MS}=866 \mathrm{~g} \text { pour } 1000 \text { de produit } \\
& \mathrm{MO}=906 \mathrm{~g} \text { pour } 1000 \mathrm{de} \mathrm{MS} \\
& \mathrm{MA}=206 \mathrm{~g} \quad-\quad \\
& \mathrm{MG}=154 \mathrm{~g} \quad- \\
& \begin{array}{llll}
\text { Mcell. }=330 \mathrm{~g} & - & - \\
\text { ENA } & =207 \mathrm{~g} & - & -
\end{array}
\end{aligned}
$$

L'utilisation de la formule classique (bromatologie $\times$ coefficient de digestibilité) permet d'attribuer à cette graine de coton une valeur fourragère de 1,17 UF par $\mathrm{kg}$ de MS. 
TABLEAU $N^{\circ}$ II - Analyses bromatologiques et coefficient de digestibilitê.

\begin{tabular}{|c|c|c|c|c|c|c|c|c|c|c|c|c|}
\hline Fourrage & $\begin{array}{l}\mathrm{N}^{\circ} \mathrm{d}^{\dagger} \text { ordre } \\
\text { digestibilité }\end{array}$ & M.S. & M.M. & M. 0. & M.A.T. & M.C. & $\begin{array}{c}\text { M. } \\
\text { Ce11. }\end{array}$ & F.N.A. & $\mathrm{Ca}$ & $P$ & $\begin{array}{c}\text { Lignine } \\
\text { (1) }\end{array}$ & $\begin{array}{c}\text { Lignine } \\
\text { (2) }\end{array}$ \\
\hline $\begin{array}{l}\text { Fane d'arachide gyrobroyée : } \\
\text { Composition (moyenne ae } 5 \text { analyses) } \\
\text { Coefficient de variation } \\
\text { Coefficient de digestibilitê : } \\
\text { moyenne coefficient de variation }\end{array}$ & $\begin{array}{l}1-2-14 \\
34-35\end{array}$ & $\begin{array}{r}870,7 \\
1,8 \\
59,4 \\
2,7\end{array}$ & $\begin{array}{l}99,2 \\
33,9\end{array}$ & $\begin{array}{r}900,7 \\
3,7 \\
60,7 \\
7,3\end{array}$ & $\left\{\begin{array}{r}107,1 \\
4,1 \\
60,6 \\
3,1\end{array}\right.$ & $\begin{array}{r}15,8 \\
20,0 \\
52,2 \\
9,8\end{array}$ & $\begin{array}{r}341,8 \\
5,4 \\
45,6 \\
14,1\end{array}$ & $\begin{array}{r}441,8 \\
1,5 \\
72,8 \\
4,3\end{array}$ & $\begin{array}{l}9,2 \\
6,2\end{array}$ & $\begin{array}{r}1,2 \\
28,6\end{array}$ & $\begin{array}{r}102,9 \\
2,9\end{array}$ & $\begin{array}{r}108,2 \\
18,6\end{array}$ \\
\hline $\begin{array}{l}\text { Fane d'arachide pulvérulente : } \\
\text { Composition } \\
\text { Coefficient de digestibilité }\end{array}$ & 3 & $\begin{array}{r}888,6 \\
54,3\end{array}$ & 83,4 & $\begin{array}{r}916,6 \\
57,3\end{array}$ & $\begin{array}{r}110,9 \\
54,9\end{array}$ & $\begin{array}{l}16,7 \\
56,4\end{array}$ & $\begin{array}{r}365,5 \\
45,6\end{array}$ & $\begin{array}{c}423,5 \\
70\end{array}$ & 11 & 1,1 & 115.6 & 129,3 \\
\hline $\begin{array}{l}\text { Fane pulverrulente + farine de sorgho : } \\
\text { Composition } \\
\text { Coefficient de digestibilité }\end{array}$ & 6 & $\begin{array}{r}877,7 \\
55,5\end{array}$ & 110 & $\begin{array}{l}890 \\
63,3\end{array}$ & $\begin{array}{r}119,5 \\
55,8\end{array}$ & $\begin{array}{l}24,1 \\
60,5\end{array}$ & $\begin{array}{r}212,6 \\
41,6\end{array}$ & $\begin{array}{r}533,8 \\
81,5\end{array}$ & 7,2 & 1,7 & 80,2 & 94,7 \\
\hline $\begin{array}{l}\text { Fane gyrobroyée + graines de coton } \\
\text { Composition } \\
\text { Coefficient de digestibilité }\end{array}$ & 10 & $\begin{array}{r}856,6 \\
56,2\end{array}$ & 59,1 & $\begin{array}{r}940,9 \\
59,8\end{array}$ & $\begin{array}{r}124,8 \\
59,8\end{array}$ & $\begin{array}{l}53,6 \\
74,6\end{array}$ & $\begin{array}{r}362,6 \\
47,4\end{array}$ & $\begin{array}{r}399,8 \\
69,4\end{array}$ & 8,0 & 0,9 & 128 & 123,5 \\
\hline $\begin{array}{l}\text { Fane de haricot: } \\
\text { Composition } \\
\text { Coefficient de dugestubilitê }\end{array}$ & 12 & $\begin{array}{r}699,2 \\
62,2\end{array}$ & 109,1 & $\begin{array}{r}890,9 \\
68,3\end{array}$ & $\begin{array}{l}163 \\
76,1\end{array}$ & $\begin{array}{l}18,1 \\
49,1\end{array}$ & $\begin{array}{l}354 \\
59,1\end{array}$ & $\begin{array}{l}355 \\
73,1\end{array}$ & 25,5 & 1,5 & 104,1 & 109,4 \\
\hline $\begin{array}{l}\text { Rations d'embouche } 1973 \\
\text { Compostion } \\
\text { Coefficient de variation } \\
\text { Coeffrcient de digestibilite }\end{array}$ & $\begin{array}{r}26-29-30 \\
31\end{array}$ & $\begin{array}{r}912,5 \\
0,2 \\
51,9 \\
3,6\end{array}$ & $\begin{array}{r}47,0 \\
7,0\end{array}$ & $\begin{array}{r}952,4 \\
0,3 \\
52,8 \\
4,1\end{array}$ & $\begin{array}{c}135,0 \\
2,13 \\
59,5 \\
4,6\end{array}$ & $\begin{array}{r}74,3 \\
0,8 \\
87,7 \\
0,8\end{array}$ & $\begin{array}{r}252,2 \\
0,3 \\
17,5 \\
26,3\end{array}$ & $\begin{array}{c}490,9 \\
0,08 \\
62,3 \\
2,4\end{array}$ & $\begin{array}{r}5,5 \\
11,4\end{array}$ & $\begin{array}{l}2,8 \\
4,0\end{array}$ & & \\
\hline
\end{tabular}

Rectificatif : $\mathrm{N}^{\circ}$ d'ordre digestibilité, lire 1-2-14-34-35; avant dernière ligne, lire 28 au lieu de $26 ; 1^{\mathrm{re}}$ colonne, $5^{\mathrm{c}}$ ligne, supprimer moyenne; ajouter en dernère ligne : coefficient de variation. 


\begin{tabular}{|c|c|c|c|c|c|c|c|c|c|c|}
\hline & & 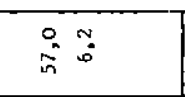 & 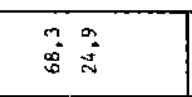 & in & 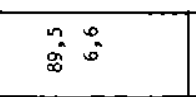 & 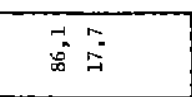 & 莗 & s. & 总 & $\begin{array}{l}0 \\
\text { sे } \\
\text { aे }\end{array}$ \\
\hline & 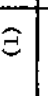 & $\hat{n}^{n}=$ & $\begin{array}{ll}* \\
n^{2} \\
n\end{array}$ & $\hat{i}$ & $\begin{array}{ll}0 \\
0^{\circ}\end{array}$ & 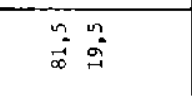 & 吕 & $\overbrace{}^{2}$ & $\stackrel{3}{=}$ & 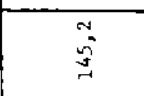 \\
\hline & 4 & $\stackrel{\infty}{=}: 0$ & $\stackrel{n}{5}$ & $\tilde{s}$ & ${ }_{i=1}^{2}{ }^{n}$ & 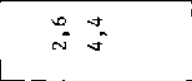 & $\stackrel{n}{0}$ & $\stackrel{\sim}{\leftrightarrows}$ & $\stackrel{\infty}{=}$ & 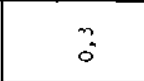 \\
\hline & ; & $a^{2}=\frac{\pi}{4}$ & ${ }_{i}^{n}=$ & $\stackrel{m}{m}$ & $\vec{m}=$ & si & $\vec{s}$ & $\stackrel{a}{;}$ & 3 & $z$ \\
\hline & 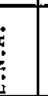 & 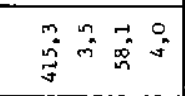 & 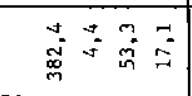 & 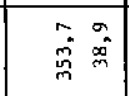 & 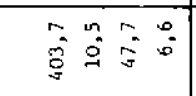 & 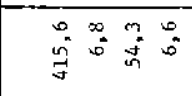 & 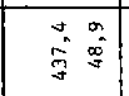 & 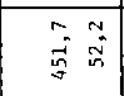 & 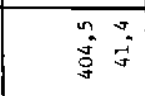 & 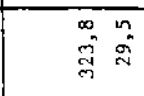 \\
\hline & $\dot{\vec{g}}$ & 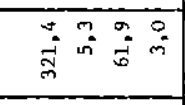 & $\begin{array}{llll}0 & 0 \\
0 & 0 \\
0\end{array}$ & $\begin{array}{c}\hat{N} \\
\tilde{n}\end{array}$ & 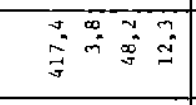 & 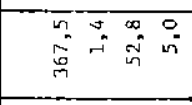 & 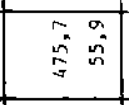 & 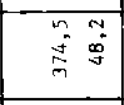 & 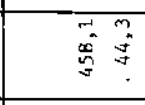 & 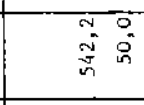 \\
\hline & 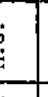 & 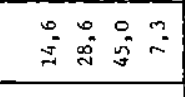 & 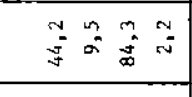 & 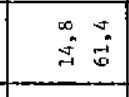 & 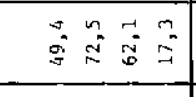 & 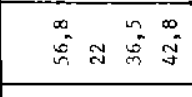 & $\begin{array}{ll}0 & 0 \\
0 & 0 \\
0 & 0 \\
\end{array}$ & 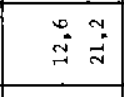 & 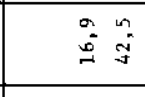 & 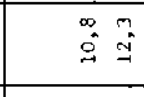 \\
\hline & 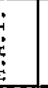 & 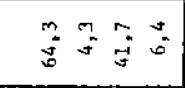 & 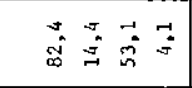 & 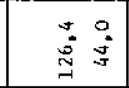 & 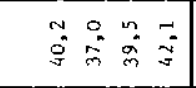 & 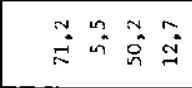 & 品 & $\begin{array}{l}* \\
0 \\
0\end{array}$ & 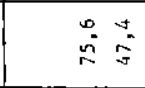 & $\begin{array}{ll}\sim \\
\cdots \\
\infty \\
\infty\end{array}$ \\
\hline & 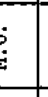 & 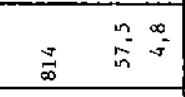 & 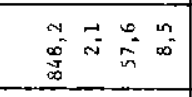 & 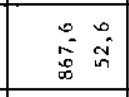 & 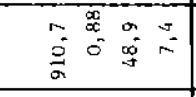 & 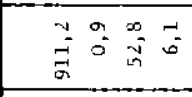 & 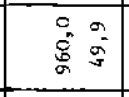 & 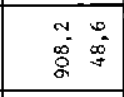 & 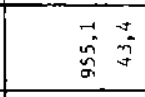 & $\begin{array}{ll}0 \\
0 \\
0 \\
0\end{array}$ \\
\hline & 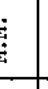 & 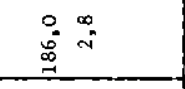 & $\begin{array}{l}=0 \\
\dot{\vec{s}} \\
\underline{\Xi}\end{array}$ & $\underset{\sim}{\tilde{m}}$ & 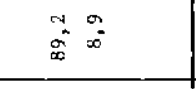 & $\begin{array}{cc}c & 0 \\
x_{0} & a^{2}\end{array}$ & $\begin{array}{l}0 \\
\dot{9} \\
\end{array}$ & $\stackrel{\infty}{+}$ & f & $\begin{array}{l}0 \\
\dot{3} \\
0\end{array}$ \\
\hline & 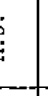 & 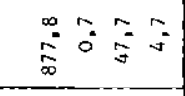 & 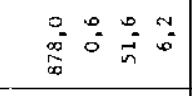 & 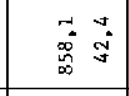 & 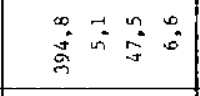 & 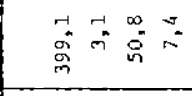 & 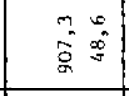 & 势 & 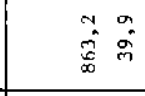 & $\begin{array}{ll}0 & 0 \\
0 \\
0 \\
0 \\
0\end{array}$ \\
\hline & 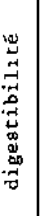 & 站 & 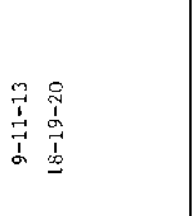 & $\infty$ & I & 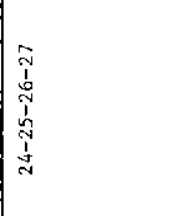 & + & $\stackrel{m}{i}$ & n & r \\
\hline & 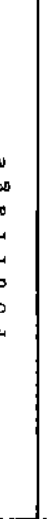 & 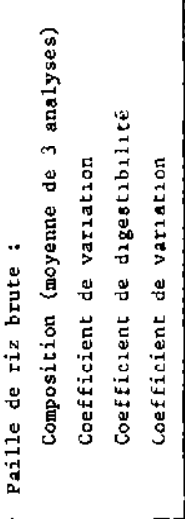 & 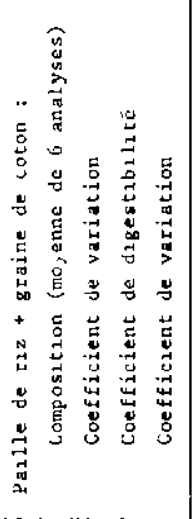 & 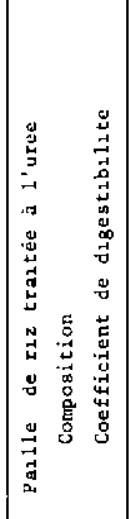 & 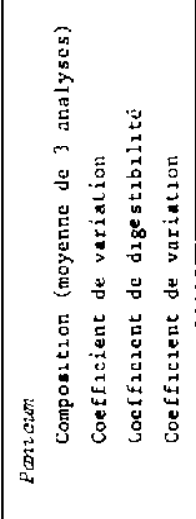 & 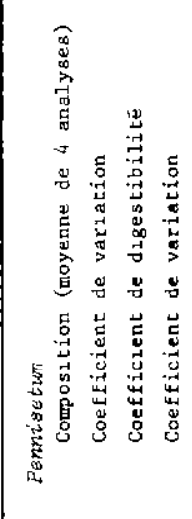 & 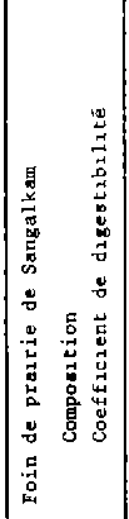 & 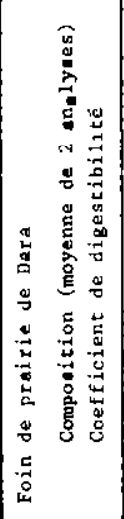 & 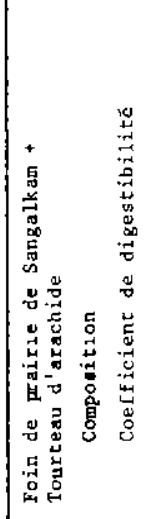 & 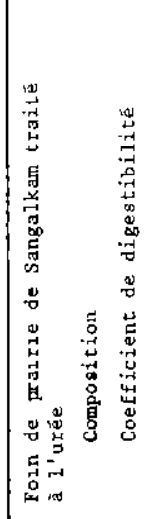 \\
\hline
\end{tabular}


TABLEAU $\mathrm{H}^{\bullet}$ IV-Valeurs fourragères des aliments utilisés Indice de consommation (I.Q.) et indice de valeur alimentaire (I-Va).

\begin{tabular}{|c|c|c|c|c|}
\hline Four $\operatorname{cog} \mathrm{e}$ & $\begin{array}{l}\text { UF/kg M.S. } \\
\text { Formule } \\
\text { classique }\end{array}$ & $\begin{array}{l}\text { UF/kg M.S. } \\
\text { Formule } \\
\text { de Breirem }\end{array}$ & $\mathrm{I} \cdot \mathrm{Q}$. & $\mathrm{I} . \mathrm{Va}$ \\
\hline Fare gyrobroyêe : (Moyenne de 5 analyses) & 0,55 & 0,49 & 103,8 & 72,3 \\
\hline Coefficient de variation & 13,7 & 23,6 & 15,9 & 38,5 \\
\hline Fane pulvérulente & 0,52 & 0,47 & 124 & 83,2 \\
\hline Fane pulvérulente + Earine de sorgho & 0,52 & 0,57 & 122,7 & 97,4 \\
\hline Fane broyêe + graine de coton & 0,66 & 0,53 & 127 & 93,2 \\
\hline Fane de haricot & 0,66 & 0,66 & 102,7 & 93,8 \\
\hline Paille de riz brute (Moyenne de 3 analyses) & 0.40 & 0,42 & 87,9 & 52.4 \\
\hline Coefficient de varıation & 12,1 & 8,7 & 2,5 & 11,0 \\
\hline $\begin{array}{l}\text { Paille de riz + graine de coton } \\
\text { (Moyenne de } 6 \text { analyses) }\end{array}$ & 0,49 & 0,43 & 86,4 & 52,4 \\
\hline Coefficient de varıation & 15,1 & 18,0 & 4,9 & 20,5 \\
\hline Paille de riz à 1 'urée (1 analyse) & 0,36 & 0,35 & 50,9 & 24,1 \\
\hline Panioum (Moyenne de 3 analyses) & 0,38 & 0,29 & 82,2 & 33,4 \\
\hline Coefficient de variation & 23,6 & 23,8 & 7,7 & 31,6 \\
\hline Pennisetwo (Moyenne de 4 analyses) & 0.45 & 0,37 & 102,2 & 57,8 \\
\hline Coefficient de variation & 15,6 & 16,7 & 14,8 & 11,9 \\
\hline Rations d'embouche 1973 (Moyennes) & 0,56 & 0,39 & 140,9 & 78,1 \\
\hline Coefficient de variation & 5,2 & $7, B$ & 15,5 & 27,8 \\
\hline Foin de prairie de Sangalkam & 0,28 & 0,33 & 66,7 & 30,4 \\
\hline $\begin{array}{l}\text { Foin de prairie de Dara (moyenne de } \\
2 \text { analyses) }\end{array}$ & 0,32 & 0,29 & 78,3 & 39,9 \\
\hline $\begin{array}{l}\text { Foun de prairie de Sangalkam }+ \\
\text { Tourteau d'arachide }\end{array}$ & 0,28 & 0,20 & 79,6 & 21,9 \\
\hline $\begin{array}{l}\text { Foin de prairie de Sangalkam tranté } \\
\text { à l'urée }\end{array}$ & 0,26 & 0,19 & 56,1 & 14,2 \\
\hline
\end{tabular}

TABLEAU $N^{*} V$ - Digestubilitê de la graine de coton : méthode différentielle.

\begin{tabular}{|c|c|c|c|c|c|c|}
\hline & M.S. & M.O. & M. A. & M.G. & M. cell. & E.N.A. \\
\hline Total distribué & 6126 & 5098 & 566 & 259 & 1979 & 2292 \\
\hline Paille distribuée & 4914 & 3999 & 315,9 & 71,7 & 1579 & 2040 \\
\hline Coton graine distribue & 1212 & 1099 & 250 & 187,3 & 400 & 252 \\
\hline Total refusé & 1003 & 750 & 62,8 & 10,33 & 261,6 & 409,8 \\
\hline Refusé coton & 0 & 0 & 0 & 0 & o & 0 \\
\hline Refusê paslle & 1003 & 750 & 62,8 & 10,33 & 261,6 & 409,8 \\
\hline Consommé en paille & 3911 & 3249 & 253,1 & 61,4 & 1317,4 & 1630,2 \\
\hline Consoumé coton & 1212 & 1099 & 250 & 187,3 & 400,0 & 252 \\
\hline Total excrêté & 2352 & 1661 & 237 & 36,7 & 655 & 732 \\
\hline Pallle excretée & 2045 & 1361 & 144 & 33,7 & 501 & 780 \\
\hline Coton excrété & 307 & 300 & 93 & 3 & 154 & 0 \\
\hline Coton retenu & 905 & 799 & 157 & 184 & 246 & 252 \\
\hline $\begin{array}{l}\text { Coefficrent de digestrbilité } \\
\text { de la graine de coton }\end{array}$ & 74,6 & 72,7 & 62,8 & 98,3 & 61,5 & 100 \\
\hline
\end{tabular}




\section{IV.2.2. Digestibilité différentielle du tourteau de "béref"}

Le « béref » est le nom local donné à certaines variétés de pastèques cultivées dans la région Nord du Sénégal, dont les graines grillées font l'objet d'une consommation humaine importante. Récemment, les huileries de Dakar se sont intéressées à ce produit et en ont extrait une huile aux qualités prometteuses. Le résidu de cette fabrication est un tourteau dont la vocation naturelle est l'alimentation du bétail.

Les dernières digestibilités envisagées dans cette note ont pour but en utilisant une méthode différentielle d'étudier la valeur alimentaire de ce tourteau.

Cette étude a porté sur deux types de rations : la première à base de son de maïs et la deuxième de foin de Panicum maximum. Concernant la première, une difficulté a consisté à composer une ration correctement consommée et contenant une proportion suffisante de tourteau.
Après plusieurs essais, les formules retenues ont été les suivantes:

Son de maïs $\ldots \ldots \ldots \ldots \ldots \ldots \ldots 60$

Tourteau de « béref $» \ldots \ldots \ldots \ldots . . . . .38$

Sel ........................ 2

Son de mais $\ldots \ldots \ldots \ldots \ldots \ldots \ldots . \ldots 9$

Sel ...................... 2

Quant à la ration à base de Panicum, le tourteau est distribué séparément à raison de $200 \mathrm{~g}$ par jour et par animal, sous la forme d'une suspension aqueuse administrée de force aux animaux.

Les résultats généraux concernant ces digestibilités différentielles sont consignés dans les tableaux VI et VII.

Les calculs conduisant aux coefficients de digestibilité du tourteau "béref " sont donnés dans les tableaux VIII et VIII bis. Il est à noter que dans le cas de la digestibilité n ${ }^{\circ} 37$, c'est par le calcul que les proportions de son et de tourteau ont été déterminées dans le refus

TABLEAU N ${ }^{\circ}$ VI - Digestibilités différentuelles - son de maís + rourteau de "béref" et

- Panicum + tourteau de "béref"

\begin{tabular}{|c|c|c|c|c|c|c|c|c|c|c|c|}
\hline \multirow{5}{*}{ Bromatologie } & Nature ration & $\mathrm{N}^{0}$ & M.S. & M.M. & M.O. & M.A. & M.G. & M.cell. & E.N.A. & $\mathrm{P}$ & $\mathrm{Ca}$ \\
\hline & Son de mä́ & 40 & 886,6 & 34.4 & 965,6 & 88,9 & 117 & 118,0 & 623,5 & 0,5 & 4,9 \\
\hline & $\begin{array}{l}\text { Son de mais + } \\
\text { tourteau de béref }\end{array}$ & 37 & 907,9 & 60,3 & 939.7 & 217 & 111,7 & 179,5 & 431,5 & 0,9 & 6,6 \\
\hline & Panicum marimum & 41 & 854,5 & 99.8 & 900,2 & 92,7 & 80,6 & 403,5 & 323,4 & 3,74 & 1,61 \\
\hline & $\begin{array}{l}\text { Panicum maximum + } \\
\text { courteau de bëref }\end{array}$ & 42 & 861,2 & 95.7 & 904,3 & 128,2 & 81,5 & 394,2 & 297,5 & 3,41 & 2,30 \\
\hline \multirow{4}{*}{$\begin{array}{l}\text { Coefficients } \\
\text { de } \\
\text { digestibilité }\end{array}$} & Son de maîs & 40 & 60,2 & & 63,3 & 40,9 & 47,4 & 74,1 & 67,2 & & \\
\hline & $\begin{array}{l}\text { Son de mais }+ \\
\text { tourteau de béref }\end{array}$ & 37 & 67.7 & & 69.4 & 76 & 84,2 & 40,3 & 73,6 & & \\
\hline & Ponicum maximom & 41 & 49,4 & & 50,7 & 61,6 & 65,0 & 53,1 & 40,4 & & \\
\hline & $\begin{array}{l}\text { Ponicum maximum + } \\
\text { tourteau de béref }\end{array}$ & 42 & 53,0 & & 54,5 & 65,9 & 65,8 & 53,3 & 45,6 & & \\
\hline
\end{tabular}

TABLEAL N VII - Valeurs fourragères et indices

\begin{tabular}{|l|c|c|c|c|}
\hline & N* & $\begin{array}{c}\text { UF/kg M.S. } \\
\text { méthode } \\
\text { Elassique }\end{array}$ & $\begin{array}{c}\text { UF/kg M.S. } \\
\text { formule de } \\
\text { Breirem }\end{array}$ & I.Q. \\
\hline $\begin{array}{l}\text { Son de maïs } \\
\text { Pconicum }\end{array}$ & 40 & 0,86 & 0,62 & 0,33 \\
\hline $\begin{array}{l}\text { Son de maïs + } \\
\text { tourteau de béref }\end{array}$ & 37 & 0,47 & 54,7 \\
\hline $\begin{array}{l}\text { Panicum + } \\
\text { tourteau de béref }\end{array}$ & 42 & 0,95 & 0,72 & 89,7 \\
\hline
\end{tabular}


TABL. $\mathbb{N}^{\circ}$ VIII-Digestibilitê diffêrentielle tourteau de "bêref". Son de maĩs ( $n^{\circ} 37$ et 40). Total distribué sec : 36344 Total distribué brut : $36344 \times 1000=40030$

\begin{tabular}{|c|c|c|c|c|c|c|}
\hline & M.S. & M.o. & M.A. & $M, G$. & M.c. & E.N.A. \\
\hline Total distribue sec & $36 \quad 344$ & 34152 & 7886 & 4059 & 6523 & 15682 \\
\hline Distribué son & $21 \quad 294$ & 20561 & 1893 & 2506 & 2512 & 13276 \\
\hline Distribué tourteau & 15050 & 13591 & 5993 & 1553 & 4011 & 2406 \\
\hline Total refusé & $20 \quad 417$ & 16987 & 4796 & 2043 & 3689 & 8458 \\
\hline Refusê son & 12719 & 11280 & 1274 & 1307 & 1484 & 7214 \\
\hline Refusé tourteau & 7698 & 7707 & 3522 & 736 & 2205 & 1244 \\
\hline Total consommé & 15927 & 15165 & 3090 & 2016 & 2834 & 7224 \\
\hline Consontué son & 8575 & 9281 & 619 & 1199 & $102 B$ & 6062 \\
\hline Consomue tourteau & 7352 & 5884 & 2471 & 817 & 1806 & 1162 \\
\hline Total excrété & $509 \mathrm{I}$ & 4610 & 695 & 324 & 1671 & 1918 \\
\hline Son excrêtế & 2769 & 2839 & 148 & 189 & 613 & 1600 \\
\hline Tourteau excrété & 2322 & 1771 & 547 & 135 & 1058 & 31.8 \\
\hline Tourteau retenu & 5030 & 4113 & 1924 & 682 & 748 & 844 \\
\hline $\begin{array}{l}\text { Coefficients de } \\
\text { digestrbilute tourteau }\end{array}$ & 68,4 & 69,9 & 77,8 & 83,4 & 41,4 & 72,6 \\
\hline
\end{tabular}

Rectificatif : $1^{\text {re }}$ lıgne du titre, lıre tourteau de béref/son de maïs.

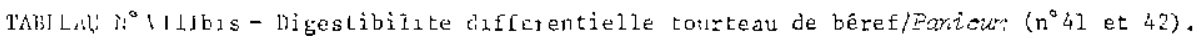

\begin{tabular}{|c|c|c|c|c|c|c|}
\hline & M.S. & $\mathrm{M}, \mathrm{O}$ & M.A. & M.G. & M.c. & E.N.A. \\
\hline $\begin{array}{l}\text { Rapport ingere/excrete à } \\
\text { partir de la digestibilité }{ }^{\circ}{ }^{\circ} 41\end{array}$ & 1,974 & 2,026 & 2,595 & 2,855 & 2,131 & 1,677 \\
\hline Excreté digeslibilite $n^{\circ} 2$ & 3325,73 & 2916,66 & 292,66 & 197,54 & 1295,03 & 1128,42 \\
\hline $\begin{array}{l}\text { Consomate Faniex m } \\
\text { digestibilute } n^{\circ} 42\end{array}$ & 5774,97 & 5165,13 & 576,91 & 496,06 & 2252,81 & 1837,59 \\
\hline Lxcrete I'onicuh calculé & 2925,51 & 2549,42 & 222,31 & 173,75 & 1057,16 & 1095,76 \\
\hline Lxcrétó tourteau $(2-4)$ & 400,22 & 367,24 & 70,35 & 23,79 & 240.87 & 32,66 \\
\hline Consommé tourteau & 1307,6 & 1216,98 & 466,02 & 114,67 & 464,19 & 172,08 \\
\hline $\begin{array}{l}\text { Coefficient digestibilite } \\
\frac{(6-5)}{6}\end{array}$ & 69,4 & 69,8 & 84,9 & 79,25 & 48,1 & 81,0 \\
\hline
\end{tabular}

Rectificatif $1^{\text {re }}$ colonne, lire Excrété digestibilité $n^{\circ} 42$; supprimer (2-4) après tourteau ; remplacer $\frac{6-5}{6}$ par tourteau.

(62,35 p. 100 de son et 36,55 p. 100 de tourteau), ce calcul se faisant à partir de la composition bromatologique du refus d'une part, du son et du tourteau d'autre part.

La valeur fourragère du tourteau de béref déduite du précédent tableau est de $0,89 \mathrm{UF} / \mathrm{kg}$ MS et MAD $=309$.

La valeur fourragère du tourteau de béref déduite de ce tableau est de $0,89 \mathrm{UF} / \mathrm{kg}$ MS et $\mathrm{MAD}=338$.
Les résultats présentés dans les deux tableaux précédents semblent donc attribuer une valeur alimentaire appréciable au tourteau de béref $(0,89$ UF et $323 \mathrm{MAD}$ au $\mathrm{kg}$ d'aliment sec), le situant presqu'au niveau du tourteau de graine de coton. Par ailleurs, le fait que deux rations différentes donnent sensiblement la même valeur à ce tourteau, rend ces résultats relativement fiables. 


\section{v. DISCUSSIONS}

\section{V.1. Fanes d'arachide et de haricot}

Les 5 digestibilités réalisées à partir de la fane d'arachide broyée font apparaître une assez grande variabilité des résultats d'une expérimentation à l'autre.

Cette variabilité se rencontre au niveau des analyses bromatologiques et porte essentiellement sur le taux des matières minérales et des matières grasses. Les coefficients de variation sont en effet pour ces deux éléments de 34 et 20 p. 100.

Elle apparaît encore dans l'expression des coefficients de digestibilité et frappe surtout ceux concernant la cellulose (coefficient de variation 14,1 p. 100 . Elle s'accentue encore dans les résultats finals : valeur fourragère et indices.

Or, les calculs intéressant les 8 digestibilités à base de fane d'arachide montrent qu'il existe une corrélation négative significative entre les taux de matières minérales et les valeurs fourragères correspondantes $(r=-0,73)$. La variabilité observée n'est donc pas liée à une déficience de la méthode mais résulte de différences dans la composition de l'aliment distribué, différences portant surtout sur le taux des matières minérales.

Ces dernières sont représentées surtout par de la silice provenant du sable incorporé à la fane au moment de la récolte et du battage.

On peut donc dire que la valeur d'une fane, en tant qu'aliment du bétail dépend, pour une grande part, de son état de propreté. Toute souillure par le sable entraine une mauvaise utilisation de la cellulose et en conséquence une diminution très sensible de la valeur fourragère.

Les résultats moyens obtenus dans ces expériences attribuent à la fane d'arachide une valeur fourragère de $0,55 \mathrm{UF}$ et $65 \mathrm{MAD}$ au $\mathrm{kg}$ de MS. Le rapport MAD/UF $=118$ est en général trop élevé pour l'alimentation des diverses espèces. La supplémentation de la fane d'arachide semble donc nécessiter l'adjonction d'un produit riche en énergie et pauvre en azote. Dans les deux essais de supplémentation réalisés avec de la farine de sorgho dans un cas, de la graine de coton dans l'autre, les meilleurs résultats sont obtenus avec le sorgho. En effet, si l'on considère les indices de valeur alimentaire qui semblent les plus représentatifs pour comparer la valeur de plusieurs rations, on obtient la progression suivante :

Fane d'arachide seule .......... 72

Fane d'arachide + graine de coton. . 93,2

Fane d'arachide + sorgho ....... 97,4

11 est à noter que le simple traitement physique de la fane, sa résolution en farine fait passer l'indice de valeur alimentaire de 72 à 83,2 .

Une dernière remarque concernant cette première série de digestibilités concerne l'intérêt alimentaire de la fane de haricot. Celle que nous avons utilisée était encore relativement verte (taux de matières sèches : 69,9 p. 100) et recelait un taux important de matières azotées (16,3 p. 100).

Elle a permis d'obtenir une valeur de 0,66 UF et de 124 MAD au $\mathrm{kg}$ de MS, caractéristiques qui rendent ce fourrage très comparable à la farine de feuilles de luzerne produite en Europe.

Les indices de consommation rapportés dans le tableau IV montrent que la fane d'arachide est un fourrage très appété par le mouton. Pour la fane seule ou pour la fane supplémentée, la valeur de l'indice se situe au-dessus de 100 .

Il ne semble pas que la souillure par le sable constitue un obstacle à la consommation.

\section{V.2. La paille de riz}

Les 3 digestibilités concernant la paille de riz seule et les 6 expériences portant sur la paille de riz supplémentée par la graine de coton tểmoignent d'une reproductibilité assez bonne.

Sur les résultats finals, la valeur fourragère par exemple, le coefficient de variation est de 12 p. 100 dans un cas et 15 p. 100 dans l'autre.

Cela tient à ce que la paille de riz utilisée s'est avérée être un fourrage homogène et relativement constant dans sa composition. Des variations sensibles apparaissent seulement au niveau des taux de matières grasses et du calcium.

Dans un précédent travail intitulé « la paille de riz dans l'alimentation animale au Sénégal ", nous avions déjà remarqué que si la paille d'une récolte est relativement constante dans sa composition, il n'en est plus de même d'une année à l'autre. Le taux moyen des matières azotées était par exemple de 21 p. 1000 sur 25 échantillons en 1963 ; de 22,8 p. 1000 sur 33 échantillons en 1965 ; de 31,2 p. 1000 sur 27 échantillons en 1970. 
TABLEAU $N^{\circ} \mathrm{IX}$ - Comparaison des digestibilitês sur bovins et sur moutons

\begin{tabular}{|c|c|c|c|c|c|c|c|c|c|c|}
\hline & & M.S. & M.M. & M.O. & M.A. & M.G. & M.cell. & E.N.A. & $\mathrm{Ca}$ & $\mathbf{P}$ \\
\hline \multirow{4}{*}{ bromatologie } & Digest.bovaris pailie 1963 & 936,7 & 175,0 & 825,0 & 21,0 & 18,6 & 361,7 & 425,6 & 1,7 & 0,69 \\
\hline & Digesc.bovins parlle 1965 & 922,7 & 179,1 & 220,4 & 22,8 & 9,8 & 345,0 & 442,6 & 1,7 & 0,65 \\
\hline & bigest.bovins paille 1970 & 891,8 & 171,9 & 828,9 & 31,2 & 13,7 & 330,4 & 453,9 & 2,15 & 0,75 \\
\hline & Ligest.moulon paille 1973 & 877.8 & 186 & 814,0 & 64,3 & 14,6 & 321,4 & 415,3 & 2,4 & 1,8 \\
\hline \multirow{3}{*}{$\begin{array}{l}\text { Coeflacient de } \\
\text { dlgestibilile }\end{array}$} & Uigest.'sovas paille 1963 & 52,7 & & 61,2 & -44 & 66,0 & 67,1 & 59,4 & & \\
\hline & Wrescolovins parle 1965 & 56,6 & & 64.6 & -11 & 67,8 & 75,0 & 58,9 & & \\
\hline & Ligest. nouton pullie 1973 & 47,7 & & 57,5 & 41,7 & 45,0 & 61,9 & 58,1 & & \\
\hline \multirow{3}{*}{$\begin{array}{l}\text { Vuleurs } \\
\text { sourrageres }\end{array}$} & Ligest.bovins paille 1963 & \multicolumn{9}{|c|}{$0.4 j$ uF et $0 \mathrm{MAD}$} \\
\hline & Drgest.bovins parlle 1965 & \multicolumn{9}{|c|}{0,47 UF et 0 MAD } \\
\hline & Digest.nouton liaslle 1973 & \multicolumn{9}{|c|}{0,40 UF et 26 MAD } \\
\hline
\end{tabular}

Rectificatif : $\ll$ M. O. $» 2^{\mathrm{e}}$ ligne lıre 820,4 .

Ce taux est aujourd'hui de 64,3 et on ne peut plus parler de la très grande indigence de ce fourrage en matières azotées comme nous l'avions fait autrefois. Ces pailles, ayant la même origine, il est fort probable que leur enrichissement progressif d'une année à l'autre en matières azotées tient à une utilisation de plus en plus intensive des engrais.

Les premières digestibilités sur la paille de riz avaient été effectuées sur bovin. Il est intéressant de comparer les résultats obtenus à l'époque avec les résultats actuels obtenus sur mouton. Les éléments de ces comparaisons font l'objet du tableau IX.

Le tableau $n^{\circ}$ IX montre que malgré la différence des pailles et également des méthodes, les résultats obtenus sur bovins et sur moutons ne semblent pas sensiblement différents.

Les nutriments essentiels de ce fourrage sont représentés par les matières cellulosiques et l'ENA.

Or, les coefficients moyens obtenus sur bovins pour ces deux éléments sont respectivement 71 p. 100 et 59,1 p. 100 tandis que sur les moutons, ils sont de 61,9 et 58,1 .

L'estimation finale pour les valeurs fourragères diffère cependant quelque peu : 0,46 UF pour les bovins et 0,40 UF pour les moutons.

Malgré l'augmentation progressive du taux des matières azotées de la paille produite au Sénégal, ce fourrage demeure encore très pauvre en azote. Il faut donc obligatoirement pour l'utiliser correctement le supplémenter avec des produits capables d'en apporter.
Dans le cadre restreint de ces expérimentations, nous avons utilisé la graine de coton et le traitement de la paille par l'urée.

Avec $200 \mathrm{~g}$ de graine de coton par animal et par jour, la valeur fourragère de la ration augmente légèrement $(0,49$ UF au lieu de 0,40$)$ de même que sa teneur en MAD (42 MAD au lieu de 26) de cette ration dont le rapport MAD/ UF parvient à 100 , ce qui est le gage d'une espérance de production favorable.

Le traitement de la paille de riz par l'urée (immersion du fourrage dans une solution à 2 p. 100 d'urée pendant $6 \mathrm{~h}$ et séchage) produit bien une augmentation sensible du taux de matières azotées du fourrage mais agit sensiblement sur la consommation dont l'indice passe de 88 pour la paille pure à 50 pour la paille traitée. Donc, ce procédé qui diminue sensiblement l'appétence de la paille ne paraît pas favorable. De meilleurs résultats auraient certainement été obtenus par un traitement à base de mélasse et d'urée.

\section{V.3. Foins de prairies naturelles}

Les essais ont porté sur deux foins de prairie naturelle en provenance de Dara et un foin fauché et fané sur les pâturages de Sangalkam. Nous rappelons la valeur alimentaire de ces fourrages.

Dara $1: 0,38$ UF et 29 MAD

Dara $2: 0,27$ UF et 16 MAD

Sangalkam : 0,28 UF et 0 MAD.

On constate que si la valeur fourragère de ces fourrages est à peu près normale, il n'est pas de même de leur valeur azotée qui est très faible si on la compare par exemple à un foin normal 
des prairies européennes (0,35 UF et 5 I MAD). Ces foins doivent donc, comme la paille de riz être supplémentés en azote.

Le traitement à l'urée appliqué précédemment à la paille de riz a été tenté sur le foin de Sangalkam avec quelques résultats. Après le traitement, la valeur fourragère passe à 0,26 UF et $43 \mathrm{MAD}$ sans une diminution notable de l'indice de consommation.

Un autre essai de supplémentation du fourrage de Sangalkam a été réalisé avec du tourteau d'arachide à raison de 6 p. $100 \mathrm{du}$ fourrage distribué.

La ration obtenue a pris alors la valeur de $0,28 \mathrm{UF}$ et $35 \mathrm{MAD}$, ce qui constitue une nette amélioration pour l'utilisation de ce fourrage pauvre.

\section{V.4. Plantes fourragères}

Les espèces testées au cours de ces digestibilités l'ont été à un stade végétatif qui ne correspond pas du tout à celui auquel doivent être exploités ces fourrages. Des travaux sont en cours sur de nouvelles digestibilités de ces plantes fourragères exploitées à 27 ou 30 jours.

Les résultats en seront présentés dans un rapport particulier.

La comparaison des deux espèces végétales, Panicum maximum et Pennisetum variété Kızozi, effectuée au même stade végétatif et avec des taux de matières sèches comparables, montre la supériorité du Pennisetum sur le Panicum (voir tableau $n^{\circ}$ IV). Il n'est d'ailleurs pas certain que cette supériorité se maintıenne au stade d'exploitation normal de ces deux fourrages.

\section{V.5. Rations d'embouche}

Les résultats obtenus au cours de ces digestibilités 0,56 UF et 79 MAD semblent sousestimer légèrement la valeur de ces rations qui ont permis d'obtenir sur des zébus un C.Q.M. de 938 durant 85 jours avec une consommation moyenne de $8,1 \mathrm{~kg}$ d'aliment par animal et par jour.

En effet, si on estime les besoins d'entretien à 2,3 UF (leur poids moyen durant l'expérience était de $212 \mathrm{~kg}$ ) et les besoins de production à $3,23 \mathrm{UF}$, la valeur journalière de la ration serait de 5,53 UF et celle d'un $\mathrm{kg}$ brut de cette ration $0,62 \mathrm{UF}$. En sec, on aurait donc une valeur fourragère de $0,67 \mathrm{UF}$.

\section{V.6. Tourteau de béref par digestibilités diffé- rentielles}

Ces digestibilités avaient pour but de déterminer par méthode différentielle la valeur du tourteau de "béref ", une digestibilité par voie directe étant rendue impossible par le fait que ce sous-produit des huileries sénégalaises, est très peu appété des animaux lorsqu'il est donné seul.

Les calculs présentés dans les tableaux VIII et VIII bis conduisent à une estimation prometteuse de ce tourteau quant aux possibilités de son utilisation pour l'alimentation du bétail.

\section{CONCLUSIONS}

Cette première série de digestibilités portant sur 39 expérimentations et six grands types de ration a montré que d'une façon générale, les digestibilités in vivo sur mouton conduisent à des résultats satisfaisants.

La variabilité observée dans le cadre d'une ration et d'une expérience à l'autre provient essentiellement de l'hétérogénéité de l'aliment distribué et non du comportement des animaux ou de l'insécurité des analyses chimiques.

Telle quelle, cette méthode, d'autant plus valable que la ration est de composition simple et constante, constitue un moyen de travail utile pour la connaissance des divers aliments utilisés ou utilisables dans l'alimentation des animaux au Sénégal.

\section{SUMMARY}

Tropical cattle nutrition under grazing range conditions in the Sahel : measurement of the dry matter intake and estimation of digestibility and feeding value of forages. II. Note dealing with the results of a first series of " in vivo digestibilities" on sheep

Authors report the results of 39 " in vivo digestibility " experiments on sheep. Different types of rations were tested: ground-nut, bean and rice straw, various hays and forages, and compound rations. 
The results obtained show that the selected method is appropriate and particularly recommanded when the ration involved offers a simple and constant composition.

\title{
RESUMEN
}

\begin{abstract}
Nutrición de los bovinos tropicales en ganaderias extensivas sahelianas : medidas de consumo y valoración de la digestibilidad y del valor alimenticio de los forrages. II. Nota concerniendo a los resultados de una primera serie de digestibilidades in vivo sobre oreja
\end{abstract}

Los autores dan los resultados de 39 ensayos de digestibilidad in vivo sobre oveja de diferentes tipos de raciones : hojarascas de cacahuete y de judias, paja de arroz, varios henos y forrajes, asi como raciones compuestas. Según estos resultados, este método de valoración de la digestibilidad es satisfactorı y tanto más eficaz cuanto que la ración experimentada tiene una composición simple y constante.

\section{BIBLIOGRAPHIE}

1. CALVET (H.), VALENZA (J.), BOUDERGUES (R.), DIALLO (S.), FRIOT (D.) et CHAMBON (J.). La paille de riz dans l'alimentation au Sénégal. Rev. Elev. Méd. vét. Pavs trop., 1974, 27 (2) : 207-221.

2. CRAMPTON (E. W.), DONEFER (E.) et LLOYD (L. E.). A nutritive value index for forages. J. anim. Sci., 1960, 19 : 538-544, 545-552.

3. DEMARQUILLY (C.). (a) Valeur alimentare de l'herbe des prairies temporaires aux stades d'exploitation pour le pâturage. JI. Quantité Ingérée par les vaches laitières. Annls Zootech., 1966, 15 (2) : 147169.

4. DEMARQUILLY (C.). (b) Valeur alimentaire de la luzerne : fourrages, $1966,26: 12-33$.

5. DLMARQULLLY (C.) el WEISS (Ph.). Tableaux de la valeur alimentaire des fourrages SEI. Ministère de l'agriculture I. N. R. A., 1970 Etude n ${ }^{\circ} 42$.

6. ELLIS (G. M.), MATRONE (G.) et MAYNARD (L. A.). A 72 p. $100 \mathrm{H}_{2} \mathrm{SO}_{4}$ method for the determination of lignin and its use in animal nutrition studies. J. anim. Sci., 1946, 5 : 286.

7. KANE (E. A.), JACOBSON (W. C.), ELY (R. E.) et MOORE (L. A.). The estimation of the dry matter consumption of grazing animals by ratio techniques. J. dairy Sci., 1953, $36: 637$.

8. LAMBOURNE (L. J.) et REARDON (T. F.). The use of chromic oxide and fecal nitrogen concentration to estimate the pasture intake of Merino wethers. Aust. J. agric. Res., 1963, $14: 257$.

9. LOCHER (E.). Estimation de la valeur alimentaire des fourrages grossiers à partir de moutons en cages de métabolisme. Rev. Suisse agric., 1972, 4 (1) : 24-29.

10. OLUBAJO (F. O.), VAN SOEST (P. J.) et OYENUGA (V. A.). Chemical composition of tropical grasses by the detergent methods and their in vitro digestibility. Conference on agricultural research and production in Africa, sept. 1971. J. Ass. Avanc. Afr. Sci. Agric., 1973, 2 (suppl. 1 August.) : 135-138.
11. OSBOURN (D. F.), THOMSON (D. J.) et TERRY (R. A.). Proc. Ioth int. Grassld Congr. Helsinki, 1966, pp. 363-366.

12. OSBOURN (D. F.). The intake of conserved forages. Proc. Symp. on fodder conservation. Reading Univ., Jan. 2-6, 1967. Occas. Symp. Br. Grassld Soc., 1967 (3) : $20-28$

13. TILLEY (J. M. A.) et TERRY (R. A.), A two stage technique for the in vitro digestion of forage crops. f. Brit. grassland Soc., 1963, $18: 104$.

14. VAN SOEST (P. J.). Use of detergents in the analysis of fibrous feeds. 1) Preparation of fiber residues of low nitrogen content $J . A . O . A . C ., 1963,46,5$ : 825.

15. VAN SOEST (P. J.). Use of detergents in the analysis of fibrous feeds. 2) A rapid method for the determination of fiber and lignin $J . A . O . A . C ., 1963$, 46, $5: 829$.

16. VAN SOEST (P. J.), MOORE (L. A.). New chemical methods for analysis of forages for the propose of predicting nutritive value. Proc. 9th. Int. Grassland Congr., São Paulo, Brazil, 1965, paper 424.

17. VAN SOEST (P. J.). Non nutritive residues: a system of analysis for the replacement of crude fiber. J. A. O. A. C., 1966, 49 (3) : 546.

18. VAN SOEST (P. J.). Development of a comprehensive system of feed analysis and its application to forages. J. anim. Sci., 1967, $26: 119-128$.

19. VAN SOEST (P. J.) et WINE (R. H.). Use of detergents in the analysis of fibrous feeds. 4) Determination of plant cell wall constituents. J. A. O. A.C., 1967, 50: 50 .

20. VAN SOEST (P. J.) et WINE (R. H.). Determination of lignin and cellulose in acid-detergent fiber with permanganate $J . A . O . A . C ., 1968,51$ (4) : 780.

21. WILSON (A. D.), WEIR (W. C.) et TORELL (D.T.). Comparison of methods of estimating the digestibility of range forage and browse. J. anim. Sci., 1971, $32,5: 1046$. 